\title{
7th meeting of the Global Arthritis Research Network
}

\author{
Matthias Brock, Michelle Trenkmann, Maria Filkova, Hossein Hemmatazad, Emmanuel Karouzakis, Fabienne Niederer, \\ Kerstin Klein and Steffen Gay*
}

\begin{abstract}
Last October, the 7th meeting of the Global Arthritis

Research Network was held in Zurich, Switzerland.

European and American experts who have made major

recent contributions to molecular biology got together

to provide insights into novel technologies and

approaches useful for biomedical research, especially

for research on arthritis and related conditions.
\end{abstract}

\section{Introduction}

The purpose of Global Arthritis Research Network (GARN) is to promote and encourage scientific interactions and collaborations that facilitate the progress of basic and clinical research in arthritis and related conditions. Last October, the 7th meeting of GARN was held in Zurich, Switzerland. The program - organized by Steffen Gay and Peter E Lipsky, associate editor and editor-in-chief, respectively, of Arthritis Research $\mathcal{E}$ Therapy - was designed to provide the attendees with the newest insights into molecular biology related to musculoskeletal diseases. The opening session, entitled 'Genetics - epigenetics - proteomics', was followed by sessions on 'Signaling pathways', 'Molecular immunology', 'Cellular autoimmunity', and 'Cell biology'. The final session, on 'Updates in rheumatology', ended with an outlook on novel modulators of pain. During the breaks and social gatherings, students and young fellows had the opportunity to ask questions and discuss the novel aspects of advanced technologies in molecular research. Fellows from the Center of Experimental Rheumatology at the Department of Rheumatology at the University Hospital in Zurich prepared notes from each session.

*Correspondence: steffen.gay@usz.ch

Department of Rheumatology, University Hospital, Gloriastrasse 25, $\mathrm{CH}-8091$ Zurich, Switzerland

\section{Session 1: Genetics - epigenetics - proteomics (part 1) \\ Chairs: Ravinder N Maini, London, UK, and Astrid Jüngel, Zurich, Switzerland}

\section{Session 1, part 1 summarized by Matthias Brock}

\section{Novel genotyping technologies}

loannis Ragoussis, Oxford, UK

Dr. Ioannis Ragoussis's presentation focused on novel genotyping technologies for the identification of diseaseassociated changes in DNA sequences. He began his talk by giving an overview of the role of single nucleotide polymorphisms in human diseases and the currently available DNA sequencing platforms. Since the analysis of a larger number of patients is hindered by limitations of currently available technologies, Dr. Ragoussis presented a new sequencing platform. This novel genotyping technology is based on an Affymetrix genotyping array (Affymetrix, Santa Clara, CA, USA) combined with DNA ligation steps allowing the sequencing of a large number of samples. To illustrate this improved sequencing method, Dr. Ragoussis presented data from his lab, showing newly identified copy number variation events in patients with autism. In the last part of his talk, he discussed the interesting field of transcriptome analysis. He explained that RNA quantification by sequencing combined with chromatin immunoprecipitation offers a powerful new tool for studying the regulation of gene expression. He also mentioned how microRNA (miRNA) quantification studies followed by RNA transcriptome analysis (miRNA-RNA Seq) could be used to understand the widespread effect of miRNAs on the RNA transcriptome.

\section{Single-gene genetic analysis Ernst Hafen, Zurich, Switzerland}

In his talk, Dr. Ernst Hafen turned to the question of how cell, organ, and body size is genetically controlled. He explained that understanding the mechanisms that control organismal size is of fundamental biological and clinical importance. By performing genetic screens that 
allow a randomized introduction of mutations into the genome of Drosophila melanogaster, his lab was able to identify novel genes associated with body size. In summary, the investigators found approximately 60 genes that promoted or inhibited growth. The majority of these genes encode proteins that are part of the insulin/TOR signaling pathway. Furthermore, they could show that the activity of this pathway influences the cellular and organismal growth of Drosophila flies. In future projects, his group will perform large proteomic approaches to further characterize the function of these genes associated with body size. In the last part of his talk, Dr. Hafen discussed the influence of environmental conditions on growth and survival. He illustrated the role of the tumor suppressor Pten in caloric restriction (CR). The reduction of Pten activity, as he explained, is sufficient to compensate impaired insulin signaling and thus represents a growth advantage under starvation. To understand the complex role of Pten activity in organismal growth, his lab will employ a combination of single-gene analysis with quantitative measurement of protein levels (mass spectrometry).

\section{Functional analysis of mammalian genomes Joel Schick, Munich, Germany}

Dr. Joel Schick began his presentation with the statement that human and mouse genomes are highly conserved and comprise approximately 20,000 coding genes. This high degree of similarity between the human and murine genomes can be used to study human diseases in mice. In this regard, the detailed characterization of each murine gene and its function is essential. A powerful tool for studying gene function is the specific silencing of genes (knockout) by applying conditional gene-trapping and gene-targeting methods. The goal of Dr. Schick's group and their partners (in the European Conditional Mouse Mutagenesis Program) is to generate a collection of up to 13,000 mutated genes in mouse embryonic stem cells. This library will enable mouse mutants to be established worldwide in a standardized and cost-effective manner, making mouse mutants available to a much wider biomedical research community. In his talk, Dr. Schick explained different methods for knocking out gene expression in mouse embryonic cells. For instance, he mentioned gene trap vectors, a class of promoter-less reporter gene cassettes that integrate randomly into a large collection of chromosomal sites. It has been shown that gene trap mutagenesis is the method of choice for large-scale, genome-wide mutagenesis. Furthermore, Dr. Schick illustrated the use of the so-called Cre-Lox recombination system to generate a conditional knockout animal; in this system, a gene is knocked out only in a specific tissue or at a specific time. Cre-Lox recombination involves the targeting of a specific sequence of
DNA and splicing it with the help of an enzyme called Cre recombinase. At the end of his talk, Dr. Schick mentioned established repositories for the collection and distribution of relevant mutant strains essential for basic biomedical research.

\section{Session 1: Genetics - epigenetics - proteomics (part 2) \\ Chairs: David Pisetsky, Durham, NC, USA, and Caroline Ospelt, Zurich, Switzerland}

\section{Session 1, part 2 summarized by Michelle Trenkmann}

\section{Aggregating proteins}

Adriano Aguzzi, Zurich, Switzerland

Are prions and prionoids a more common underlying principle of human diseases than appreciated until now? In the first part of his presentation, Adriano Aguzzi raised the question of whether not only the 'bona fide' prion disease caused by the prion particle $\mathrm{PrP}^{\mathrm{Sc}}$ but also other disorders such as Alzheimer's disease or AA amyloidosis should be considered prion diseases. The pathobiological background of this view is the following: a protein can exist in two states: the normal state and the misfolded prion state. A pathogenic event initiates an assembly of misfolded proteins into aggregates, thus leading to the progressive neurologic degeneration seen in patients. $\mathrm{PrP}^{\mathrm{Sc}}$ is transmissible and follows an infectious life cycle; that is, it acts as a seed to recruit more $\mathrm{PrP}^{\mathrm{Pc}}$. Diseases such as Alzheimer's disease or AA amyloidosis have a similar pathogenesis and are caused by the deposition of misfolded proteins (amyloid- $\beta$ and amyloid A, respectively). Aguzzi attested that AA amyloidosis has the 'best chances' of becoming a prion disease. He pointed out that prions may even have conserved 'good' properties that may be the reason that prion formation was not simply eliminated by evolution. In the second part of the presentation, Aguzzi concentrated on his own group's recent work, which deals with the question of how to study the neurotoxicity of $\mathrm{PrP}^{\mathrm{Sc}}$. They developed an ex vivo assay using mouse cerebrellar slices that could be cultured for several weeks and infected with prions. In the slices, as in the living animal, prion infection induced neurodegeneration. This technique allows investigators to address neurotoxicity questions that are difficult to study in vivo. With this model, different substances were tested for their neuroprotective activity, and work on how prion degradation could be influenced is ongoing.

\section{Novel targeting with DARPins \\ Andreas Plückthun, Zurich, Switzerland}

Andreas Plückthun started his presentation with the question 'Antibodies in inflammatory diseases: is it all 
done?' - to which he answered 'no', pointing out that approximately $30 \%$ of the patients do not respond to antitumor necrosis factor (anti-TNF) treatment. Furthermore, opportunistic infections and enormous costs are common problems of biological treatments of today. After providing a short historical overview ranging from the first monoclonal antibodies to recombinant antibodies and synthetic antibodies, he concentrated on a class of molecules - called DARPins (designed ankyrin repeat proteins) - that may substitute antibodies. These molecules are assembled from a variable number of ankyrin repeat domains that contain several randomized positions, making it possible to generate libraries of DARPins with a multitude of different binding surfaces. Their unique properties - namely these molecules are extremely well expressed, soluble, stable, and nonaggregating - and the possibility of carrying out the whole selection process in vitro by ribosomal display make them highly interesting for therapeutic approaches and research. Discussing the potential use of DARPins in therapy, Plückthun summarized work done to achieve high stability, affinity, and the ability to penetrate cells. Furthermore, he introduced a new concept of targeted gene therapy which is in development at the moment. In this therapy, adenoviruses carrying the therapeutic nucleic acid 'find' their particular target cells with the help of a specifically selected DARPin adapter. Finally, coming to applications in research, he pointed out the limitations of using DARPins in proteomics and systems biology and briefly introduced a new approach for designing modular peptide-binding scaffolds with the vision of achieving peptide recognition with predesigned building blocks of engineered Armadillo repeat proteins.

\section{Modification of histones \\ Udo Oppermann, Oxford, UK}

To begin with, Udo Oppermann gave some background information on nucleosome structure and histone modifications and their relevance to the immune system. He continued with the presentation of the Structural Genomics Consortium (SGC), a public-private partnership that aims to place protein structures of relevance to human health into the public domain and thus to promote drug discovery. Based on the SGC, the Chemical Probe Consortium works on the development of probes against specific biological targets which are then made available to the public. To concentrate on some of the possible 400 epigenetic targets, they chose bromo and tudor domains, which 'read' acetyl and methyl marks, respectively, and lysine demethylases. He presented recent progress on two targets in particular. One work was concerned with the development of a small-molecule competitor of the bromodomain BRD4, which is of interest for immunologic research since it binds to acetylated nuclear factor-kappa-B (NF-kB) and thus functions as a coactivator. The second work dealt with the search for small-molecule inhibitors of Jumonji proteins (lysine demethylases for histones and other non-histone proteins). For this, they started from $\alpha$-ketoglutarate, a cosubstrate for the demethylation process. In the end, they came up with a specific inhibitor of Jmjd3, a lysine demethylase that has already been implicated in immunerelated processes. Oppermann stated the prospect that the molecule would be publicly available in the future as soon as it has been validated in a cell-based system.

\section{DNA methylation alterations and autoimmune diseases Esteban Ballestar, Barcelona, Spain}

DNA methylation is the most common epigenetic modification and involves the 5'-methylation of cytosine in CG dinucleotides that usually reside in so-called CpG islands. Esteban Ballestar spoke about aberrant DNA methylation found in diseases ranging from cancer to genetic defects concerning epigenetic factors (for example, Rett syndrome or ICF [immunodeficiency, centromeric region instability, and facial anomalies] syndrome) and imprinting disorders such as Angelman and Prader-Willi syndromes. He pointed out that under normal conditions DNA methylation plays an important role in X chromosome inactivation, imprinting, and the regulation of tissue-specific gene expression. Therefore, promoter $\mathrm{CpG}$ islands tend to be demethylated whereas DNA repeat regions are normally methylated. In the diseased state, this seems to be reversed to a certain degree. Although cancer cells show a global hypomethylation, specific gene promoters, mainly those of tumor suppressor and DNA repair genes, show a local hypermethylation that leads to the silencing of these genes. Ballestar concluded that, also in autoimmune diseases, epigenetic influences are indicated. Having a special interest in epigenetic differences between monozygotic (MZ) twins, in whom global epigenetic differences were shown to accumulate with age, his group generated DNA methylation profiles of MZ twins discordant for autoimmune diseases such as systemic lupus erythematosus (SLE), rheumatoid arthritis (RA), and dermatomyositis. Significant differences could be detected only for SLE discordance, and many of the affected genes had an immunologic function and were of relevance in lupus pathogenesis. Hypomethylation of several genes was validated by bisulfite pyrosequencing (for example, IFNGR2 and MMP14). In contrast, DNA methylation of repetitive sequences such as LINE1 or Alu repeats was not affected. Finally, he put forward the idea that there may be an active demethylation of pathogenetically relevant genes in lupus. In the end, Ballestar presented a second study demonstrating an association between Epstein-Barr virus infection and DNA methylation in B cells. 
Session 2: Signaling pathways Chairs: Joachim Kalden, Erlangen, Germany, and Gyorgy Nagy, Budapest, Hungary

\section{Session 2 summarized by Maria Filkova}

\section{The inflammasome}

\section{Jürg Tschopp, Epalinges, Switzerland}

At the beginning of his talk, Dr. Tschopp introduced the inflammasomes as cytosolic multiprotein complexes that can proteolytically activate caspase- 1 and this subsequently leads to maturation and secretion of active interleukin IL-1 $\beta$ and IL-18. The innate immune system is characterized by its ability to recognize a wide range of pathogen-associated molecular patterns (PAMPs) (such as fungi, protozoa, bacteria, or viruses) and sterile danger-associated molecular patterns (DAMPS) (such as DNA, ATP, crystals, asbestos, $\beta$ amyloid, and cholesterol). PAMPs are recognized by pattern-recognition receptors that include the Toll-like receptors, NOD-like receptors (NLRs), C-type lectin receptors, and several other receptors. However, NLR proteins can also detect DAMPs and their recognition leads to the activation of the inflammasome. NLR-related pathways (NALP or NLRP, IPAF, and AIM2) are associated with chronic non-infectious inflammatory diseases. Dr. Tschopp presented the role of the inflammasome in the pathogenesis of several chronic inflammatory conditions.

Gout is associated with the deposition of monosodium urate (MSU) crystals. It was shown that molecular mechanisms underlying the inflammation initiated by MSU activate the NALP3 inflammasome. The involvement of IL-1 $\beta$ in this inflammatory cascade might explain the success of IL-1ra, IL-1Trap, and IL-1 $\beta$ antibody treatment in gout inflammation.

NALP3 inflammasome is implicated in the pathogenesis of another chronic inflammatory state: type 2 diabetes mellitus (DMT2). Reactive oxygen species (ROS) generation is necessary for NALP3 activation in DMT2. Moreover, when intracellular ROS increases, thioredoxininteracting protein (TXNIP) is released from the thioredoxin complex. The oxidoreductase thioredoxin is then able to act as an ROS scavenger and then free TXNIP interacts with NALP3. Thus, TXNIP is a ligand for NLRP3, leading to elevated IL-1 $\beta$ production. IL-1 $\beta$ has a pathogenic role in islet dysfunction and is a risk factor for the development of DMT2 in humans. Mouse models and human clinical trials suggest that IL-1 $\beta$ antagonism may be a promising treatment for DMT2. Finally, Dr. Tschopp presented an interesting effect of interferon-beta (IFN- $\beta$ ) on inhibition of NALP3 signaling in multiple sclerosis.

\section{Cofactor-specific regulation of NF-kB-dependent gene expression \\ Michael Hottiger, Zurich, Switzerland}

Dr. Hottiger discussed mechanisms underlying NF-kB activity and emphasized the effect of cofactors on regulation of NF-kB-dependent gene expression. The NF-kB family consists of five complexes that have an important function in regulating immune and inflammatory responses, apoptosis, cell proliferation, differentiation, and tumorigenesis. NF- $\mathrm{kB}$-specific activity depends on the preferential recruitment and localization of NF- $\mathrm{kB}$ homodimers/heterodimers, on the interaction with chromatin that enables its either constitutive and immediate or regulated and late accessibility, and on the combination of cofactors and post-translational modification of NF- $\mathrm{kB}$ dimers.

Dr. Hottiger showed that the p65 subunit of NF- $\mathrm{kB}$ is acetylated in vitro and in vivo at three different lysines K310, K314, and K315 - by the histone acetyltransferase p300. Although general transcriptional activity of RelA/ p65 was not affected, there are specific sets of genes that were upregulated or downregulated by lysine-specific RelA/p65 acetylation. SIRT-2 interacts with p65 in cytoplasm and contributes to NF- $\mathrm{kB}$-dependent gene expression via deacetylation of lysine K310. Poly (ADP-ribose) polymerase-1 (PARP-1) can also act as a promoterspecific coactivator of NF- $\mathrm{kB}$. The effect of PARP-1 is independent of its enzymatic activity but its direct interaction with both subunits of NF- $\mathrm{kB}$ is required for its coactivator function. Moreover, it was shown that acetylation of PARP-1 at specific lysine residues mediated by p300/CREB-binding protein is necessary for the abovementioned interaction of PARP- 1 with NF-kB and coactivation of NF- $\mathrm{kB}$ by $\mathrm{p} 300$. CARM1 (coactivatorassociated arginine methyltransferase) is another transcriptional coactivator of NF-kB. CARM1 forms a complex with p300 and NF- $\mathrm{kB}$ (interacts directly with the NF- $\mathrm{kB}$ subunit p65) and thus, together with the transcriptional coactivators p300/CREB-binding protein, regulates a subset of NF-kB-dependent genes. PRMT1 (protein arginine methyltransferase 1 ) synergistically coactivates NF- $\mathrm{kB}$-dependent gene expression in a complex with the transcriptional coactivators p300/CREB-binding protein, CARM1, and PARP1. In the final part of the talk, Dr. Hottiger presented the data on a mouse model of enterocolitis induced by Salmonella typhimurium and showed that PARP1 is expressed in the proliferative zone of cecum crypts, where it is required for the efficient expression of proinflammatory genes. In conclusion, cooperation between cofactors p300, PARP1, CARM1, and PRMT1 regulates NF- $\mathrm{kB}$-dependent gene activation in vivo. 


\section{Modulation of the immune response by exosomes Paul Robbins, Pittsburgh, PA, USA}

In the introduction to his talk, Dr. Robbins described exosomes as nanovesicles (40 to $100 \mathrm{~nm}$ ) generated by different cell types: reticulocytes, immune cells, platelets, epithelial cells, tumor cells, and so on. Exosomes contain antigen-presenting molecules $\mathrm{MHC}$ (major histocompatibility complex) I and MHC II and adhesion, regulatory, and costimulatory molecules and nucleic acids, including miRNAs.

Dr. Robbins demonstrated that exosomes derived from dendritic cells (DCs) treated with immunomodulatory cytokines (IL-10 and IL-4) inhibit inflammation in a murine model of delayed-type hypersensitivity (DTH). Moreover, systemic administration of exosomes derived from IL-10-treated DCs is able to suppress the onset of murine collagen-induced arthritis (CIA) as well as to reduce the severity of established arthritis.

The ability of the modified DCs expressing IL-4 and exosomes derived from these DCs to suppress the DTH response is MHC class II- and Fas-ligand/Fas-dependent. Costimulatory molecules, such as B7-1/2 and PD-L1/2, play an important role in the function of antigen-presenting cells (APCs). Lack of B7-1 or B7-2 on DCs results in a loss of the ability of IL-10-treated DCs and their exosomes to suppress the DTH response, whereas IL-10-treated DCs expressing no PD-L1/2, as well as their secreted exosomes, have no ability to suppress DTH responses. Thus, B7-1 and B7-2, but not PD-L1 and PD-L2, on IL-10-treated DCs and derived exosomes are required for immunosuppressive functions of both DCs and exosomes.

As mentioned above, exosomes released by APCs express MHC, adhesion, and costimulatory molecules and may function as antigen-presenting particles. It was presented that exosomes first bind to the APC surface and then are internalized by DCs. Thus, these internalized exosomes can be subsequently processed by DCs for presentation to $\mathrm{T}$ cells. Dr. Robbins showed that exosomes derived directly from plasma are immunosuppressive. The anti-inflammatory effect of plasmaderived exosomes in immunized mice was mediated by exosomes containing MHC II, FasL, and CD11b. Moreover, TNF $\alpha$ was shown to be bound on the membrane of exosomes derived from mouse models of CIA and from plasma of patients with RA. Therefore, there is a question of exosomes as a biomarker of the disease.

In conclusion, the immunosuppressive effect of exosomes is dependent on the presence of MHC II, FasL, and B7-1 and B7-2, and exosomes may be internalized by APCs. Thus, APC-derived exosomes could be used therapeutically for the treatment of autoimmune disease and inflammatory disorders. However, further experiments are needed to elucidate in detail the structure, mechanism of action, and clinical application of exosomes.

\section{Session 3: Molecular immunology Chairs: Peter E Lipsky, Bethesda, MD, USA, and Sandra Sacre, Brighton, UK}

\section{Session 3 summarized by Hossein Hemmatazad}

\section{Human Th-17 in infection and immunity Antonio Lanzavecchia, Bellinzona, Switzerland}

Lanzavecchia started his presentation by describing the role of Th-17 cells in inducing experimental autoimmune encephalomyelitis (EAE). He showed that mice lacking CCR6, a chemokine receptor characteristic of Th-17 cells, are resistant to the induction of EAE. CCR6 binds to its ligand, CCL20, in choroid plexus and controls immune surveillance of the central nervous system. Next, he discussed the differentiation of Th-17 cells in humans and mice. In mice, the differentiation of Th-17 cells requires transforming growth factor-beta (TGF- $\beta$ ) and IL-6, whereas in humans IL-1 $\beta$ and IL-6 are needed. In humans, in contrast to mice, TGF- $\beta$ has inhibitory effects on Th-17 differentiation. Lanzavecchia explained that in mice IL-22 has been considered a typical Th-17 cytokine and is made by fully differentiated Th-17 cells. It has been linked to skin homeostasis and inflammation. Of interest, in humans, most of the IL-22-producing cells do not make IL-17. The differentiation of $\mathrm{T}$ cells producing only IL-22 is efficiently induced in naive $\mathrm{T}$ cells by plasmacytoid DCs in an IL-6- and TNF-dependent way. Lanzavecchia and colleagues are developing high-throughput methods to analyze the human memory responses. The idea is that analyzing the repertoire of $\mathrm{B}$ cells, plasma cells, and memory $\mathrm{T}$ cells may allow us to isolate monoclonal antibodies and T-cell clones to identify target antigens and the specific class of response. Finally, he showed that Candida albicans and Staphylococcus aureus both induce Th-17 differentiation, but in different ways. $C$. albicans induces Th-17 differentiation in an IL-1 $\beta$ dependent way. IL-1 $\beta$ shuts off IL-10 production, so cells make IL-17 but not IL-10. In contrast, S. aureus can induce Th-17 differentiation in an IL- $1 \beta$-independent manner. In this case, IL-10 production is retained, so these cells have less potential to induce inflammation.

\section{Break of immunotolerance lain McInnes, Glasgow, UK}

McInnes started his talk by addressing the importance of adaptive immunity in the pathogenesis of RA. He explained how difficult it is to observe the breach of endogenous tolerance in an animal model, because these models rely on aggressive and artificial self-antigen immunization and this does not allow us to assess the very early breach of immunotolerance. A new approach 
is to look at DC behavior and their phenotype in RA, and the idea is to set up an in vivo model system that can be analyzed in real time. McInnes and colleagues took DCs and loaded them up with selected antigens (collagen type II whole protein) to generate a collageninduced mouse model of arthritis. By analyzing the interactions between DCs and T cells in this in vivo model, they could demonstrate conventional DC (cDC) activation and antigen presentation associated with breach of self-tolerance, an ability of these cells to transfer autoimmunity, together with a central role for cDCs in the TNF $\alpha$-dependent development of selfreactivity and RA pathogenesis. Furthermore, he showed some data on the effect of abatacept to limit the breach of self-tolerance via effects on the generation of $\mathrm{T}$ follicular helper cells. Abatacept prevented the emergence of self-reactivity and has potential usefulness in early inflammatory arthropathies associated with autoantibody expression.

\section{The tumoricidal activity of interleukin-12 is mediated via lymphoid tissue-inducer cells \\ Burkhard Becher, Zurich, Switzerland}

In a wonderful talk, Becher presented his data on IL-12mediated repression of subcutaneous melanoma. There are many cytokines used in cancer immunotherapy, such as IFN- $\alpha$, IL-2, and IL-12. To determine the mechanisms by which IL-12 mobilizes antitumor immunity, Becher and his group generated B16.F10 mouse melanoma cell lines that continuously release a fusion protein of IL-12. They could show that IL-12 significantly reduced tumor growth in wild-type mice whereas in IL12rb2 $2^{-/-}$mice, which lack the IL-12specific receptor subunit, melanoma cells showed no reduction in growth. But how does IL-12 repress cancer? To answer this question, they did a systemic analysis of the IL-12-induced immune responses and demonstrated that tumoricidal activity of IL-12 is independent of adaptive immunity, lymphocytes, or circulating natural killer cells. By excluding a series of cell types, which were candidates for IL-12-mediated antitumor response, they observed that IL-12responsive tumor-invading cells are in fact an innate, lineage-negative leukocyte belonging to a subset of lymphoid tissue-inducer (LTi) cells expressing the natural cytotoxicity receptor NKp46. These LTi cells also produce IL-22 and IL-17, but of interest, the IL-12mediated melanoma repression seemed to be independent of these effector cytokines. IL-12-stimulated NKp $46^{+}$LTi cells alter tumor microvasculature and this leads to upregulation of adhesion molecules (such as vascular cell adhesion molecule) and subsequent tumor control.

\section{Session 4: Cellular autoimmunity}

Chairs: Jean-Michel Dayer, Geneva, Switzerland, and Temy Mok, Hong Kong, China

\section{Session 4 summarized by Emmanuel Karouzakis}

\section{Cytokine networking \\ Eddie Liew, Glasgow, UK}

Dr. Liew presented data on the function of IL-33 cytokine in sepsis and osteoclast differentiation. IL-33 is secreted during tissue injury by a variety of cell types. The receptor of IL-33 consists of ST2 and IL-1 receptor accessory protein (IL-1RAP) that is expressed on Th- 2 cells and mast cells. The evolutionary role of IL-33 is to prevent the establishment of infection. Administration of IL-33 prevents nematode infection, Coxsackie virus infections, artherosclerosis, and asthma and attenuates sepsis. Also, IL-33 can induce allergic anaphylaxis and is associated with central nervous system diseases such as Alzheimer's disease. Dr. Liew focused his presentation on the role of IL-33 in sepsis and neutrophil migration on the site of infection. His group could demonstrate that the ST2 knockout mouse during cecal ligation and puncture is more susceptible to sepsis than the wild-type mouse. Furthermore, IL-33 improves the neutrophil migration on the site of infection and prevents downregulation of CXCR2 in neutrophils during sepsis. IL-33 allows the persistent expression of CXCR2 in neutrophils by blocking the activity of GSK2. Dr. Liew claimed that IL-33 is important in human sepsis. IL-33 induces the migration of human neutrophils during inflammation. Interestingly, sepsis non-survivors have less CXCR2 in their peripheral blood neutrophils than sepsis survivors. Finally, he showed that the soluble ST2 is highly expressed in the serum of non-survivors of sepsis. His talk concluded on the role of IL-33 osteoclast differentiation. IL-33 is expressed in the joints of TNF $\alpha$ transgenic mice. IL-33 reduces bone erosion and osteoclast formation in TNF $\alpha$ transgenic mice. The expression of TRAP, NFAT, and cathepsin $\mathrm{K}$ is reduced after IL-33 treatment. ST2 knockout mice have more bone erosion than wild-type mice. In addition, IL-33 increases the number of macrophages, DCs, and neutrophils in the spleen of the mice. Interestingly, IL-33 inhibits osteoclast differentiation and promotes the development of MT2 macrophages.

\section{Genome-wide association studies for rheumatoid arthritis} Lars Klareskog, Stockholm, Sweden

Dr. Klareskog analyzed the different genetic and environmental interactions of antibodies to citrullinated protein antigen (ANCA)-positive individuals as a risk factor for RA. Different studies in RA have shown that there is a genetic risk for RA. The most important susceptibility genes are PTPN2 and HLADR shared epitope (SE). New 
genome-wide genetic studies have shown that HLADR SE and PTPN2 are more frequent in ANCA-positive cases than ANCA-negative RA cases. Dr. Klareskog emphasized that environmental exposure such as smoking can have a great impact on the genetic influence of RA. Environmental-gene interaction studies have shown that there is a great risk of RA if you are a smoker and HLADR SE-positive. Other studies have analyzed geneto-gene interaction. These studies have determined that the PTPN2 susceptibility gene, together with HLADR SE and smoking, increases the risk for RA. Dr. Klareskog created a model for smoking and HLADR SE genes which could be tested in the laboratory. Chronic exposure of lungs to smoking increases the activation of the peptidylarginine deiminase enzymes that cause the citrullination of different proteins in the lung. APCs (macrophages and DCs) that have the SE motif may bind to some of these citrullinated proteins and trigger an immune response. Activated T cells are generated and interact with B cells. In turn, the activated B cells produce ANCA antibodies that are present very early in RA. Synovial inflammation in the joint can citrullinate synovial antigens that form complexes with ANCA and bind to the Fc receptors of synovial macrophages. His talk concluded that geneticists should separate patients with RA into two different subsets and functional immune system studies should be performed in specific T-cell subsets with a specific genetic and environmental background.

\section{Dynamic regulation of chemokines and their receptors Mario Mellado, Madrid, Spain}

Dr. Mellado described a dynamic model between the chemokine receptors CXCR1 and CXCR2 with their ligand CXCL8. Chemokines are significant for lymphocyte trafficking to the site of injury. They are a large family of small proteins that signal to a G protein-like receptor. A chemokine can activate different chemokine receptors, and immune cells express different chemokine receptors, depending on the activation stage or tissue localization. Chemokines are associated with different diseases (RA, asthma, AIDS, and cancer). A better understanding of chemokine oligomerization is needed in order to identify new antagonists. Dr. Mellado studied the dimerization of chemokine receptors by using a biophysical technique called fluorescence resonance energy transfer. A specific chemokine receptor conformation in the cell membrane can have different functional properties and activate different signaling pathways. For example, heterodimers of CXCR2/CXCR5 play an important role in cell adhesion. His talk concluded with a model of chemokine receptor homodimer and heterodimer dynamics. CXCR1 and CXCR2 form an array of homodimers and heterodimers on the cell surface. During stimulation of cells with CXCL8/IL8 ligand, there is a disruption of heterodimer formation. Homodimers are stabilized and there is high rate of CXCR2 internalization.

\section{Session 5: Cell biology \\ Chairs: Robert Eisenberg, Philadelphia, PA, USA, and Rene Toes, Leiden, The Netherlands}

\section{Session 5 summarized by Fabienne Niederer}

\section{New lessons from animal models Thomas Pap, Münster, Germany}

Thomas Pap started the cell biology session by introducing the aggressive phenotype of RA synovial fibroblasts (SFs). In an in vivo experiment, RA SFs, together with human cartilage, were implanted into severe combined immunodeficient (SCID) mice. RA SFs showed an active movement to the naive cartilage, leading to a marked destruction of the cartilage. Interestingly, RA SFs, but not osteoarthritis (OA) SFs, were able to spread the disease in the SCID mice model. Next, he discussed inducible human tumor necrosis factor transgenic (hTNFtg) mice. Like the constitutive TNF transgenic mice, these mice show an RA-like arthritis, but allow the analysis of genes, the knockout of which is embryonically lethal in a TNF $\alpha$ inflammatory background. By using these mice, Thomas Pap's group wanted to address the question of whether pathogenic changes in RA are triggered and maintained by cartilage damage. They observed that the loss of proteoglycans is an important prerequisite for the attachment of SFs to cartilage in hTNFtg mice. This attachment was mediated by integrins, as confirmed by the fact that loss of $\alpha_{1} \beta_{2}$ integrin reduced cartilage destruction not only in vitro but also in hTNFtg mice. In addition, the loss of other proteoglycan-sensing molecules, such as syndecan 4, protected mice from cartilage damage in the hTNFtg mice model of RA. Furthermore, this protection was linked to reduced production of matrix-metalloproteinase 3. Pap and his group observed the upregulation of syndecan 4 in RA SFs and in hTNFtg mice and hypothesized that syndecan 4 not only is required for SF attachment but also modulates cytokine signaling in SFs by binding the key RA cytokine IL-1.

\section{Molecular biology of aging Trygve Tollefsbol, Birmingham, AL, USA}

In his talk, Tollefsbol pointed out the trade-off between a person's dream to stay young and the continuous need for CR. Nowadays, the intake of $25 \%$ to $40 \%$ less calories, including all needed nutrients, was shown to be the best way to stay young, as CR promoted longevity not only in yeasts and mice but also in non-human primates. This finding was nicely illustrated by pictures from 
calorie-restricted, young-looking (but incredible hungry) primates.

Tollefsbol connected this effect with the molecular clock of aging. Telomere shortening was shown to correlate with the age of a cell. Moreover, telomerase was found to be activated in cancer cells. The group of Tollefsbol wondered whether in vitro sugar reduction could help answer questions about human CR and aging. Indeed, glucose restriction extended the lifespan in a human embryonic fibroblast cell line (WI-38) and, moreover, induced apoptosis in a precancerous human cell line (WI-38/S). Levels of hTERT, the gene for telomerase reverse transcriptase, were found to be induced and the tumor-suppressor protein p16 to be reduced after 2 to 4 weeks of glucose restriction in the normal cell line (WI-38). In the precancerous cell line (WI-38/S) the effects were the reverse and lower levels of hTERT and higher levels of p16 were detected. Furthermore, glucose restriction was shown to result in chromatin remodeling of the promoters of the hTERT and p16 genes. In conclusion, this presentation clearly showed that glucose restriction mediates its effects by epigenetic regulation via hTERT and p16, thereby extending the cellular lifespan of normal cells or mediating cellular senescence by induction of apoptosis in precancerous cells.

\section{Tissue repair with stem cells Rocky S Tuan, Pittsburgh, PA, USA}

Tuan started his presentation by listing different treatment options for degenerative joint diseases, such as total joint arthroplasty, non-steroidal anti-inflammatory drugs, chondrocyte implantation, and cartilage transplantation, and finally put an emphasis on regenerative surgery methods. Tuan wanted to apply principles of engineering and life sciences to the regeneration of tissue and cell organs. To address this topic, he worked with human mesenchymal stem cells (hMSCs). These cells were shown to form cartilage upon TGF- $\beta$. To scale up a little piece of cartilage became a big challenge. Tuan prepared a three-dimensional scaffold made out of nanofibers by using electrospinning processes. He finally succeeded in getting cartilage pieces with diameters ranging from 1.5 to $4 \mathrm{~cm}$. Next, Tuan's working group wanted to bring them into animals. Owing to the (now) big size of the cartilage pieces, big animals (for example, pigs) were needed. The aim of the following in vivo experiments was to repair iatrogenic cartilage defects. Tuan showed that nanofibrous scaffolds seeded with hMSCs effectively repaired cartilage defects in a swine model. Furthermore, Tuan proposed that, by regulating the stem cell niche, hMSCs should be able to differentiate into osteoblasts, chondrocytes, and adipocytes and thereby lead to the desired plasticity of the joint. In a search for genes downregulated in differentiated cells and upregulated in undifferentiated cells, they found $I L-6$ as a candidate stemness gene to maintain the proliferative and undifferentiated state of hMSC stemness, an important parameter for the optimization of manipulation of hMSCs.

\section{Session 6: Updates in rheumatology Chairs: Tsutomu Takeuchi, Tokyo, Japan, and Jesús Salvador, Madrid, Spain}

\section{Session 6 summarized by Kerstin Klein}

\section{Novel pain targets}

\section{Hanns-Ulrich Zeilhofer, Zurich, Switzerland}

In his talk, Hanns-Ulrich Zeilhofer presented three novel promising targets for the treatment of chronic pain: (a) the transient receptor potential cation channel subfamily $\mathrm{V}$ member 1 (TRPV1), (b) $\mathrm{Na}^{+}$channels (Nav1.7 and Nav1.8), and (c) the nerve growth factor (NGF). TRPV1 was originally identified as a receptor for capsaicin, the main pungent ingredient in hot chili peppers. TRPV1 channels are activated upon noxious heat and mechanical damage. TRPV1 $1^{-/}$mice were still responsive to noxious heat but were insensitive to inflammatory hyperalgesia. Clinical studies for TRPV1 antagonists were promising. However, the main side effect in treated patients was hyperthermia.

$\mathrm{Na}^{+}$channels are established targets for local analgetics and lately have also been in discussion as targets for the treatment of chronic pain. The $S C N 9 A$ gene, encoding for Nav1.7, has been associated with diverse $\mathrm{Na}^{+}$channelopathies. An SCN9A mutation, prevalent in Pakistani families and leading to a premature stop codon, led to pain insensitivity of the affected individuals. Furthermore, gainof-function mutations of the SCN9A gene cause two different diseases. In patients with erythromelalgia, Nav1.7 is activated at lower potentials, whereas in patients with paramyxal extreme pain disorder, Nav1.7 channel activation is extremely prolonged.

Another group of promising drugs for the treatment of chronic pain consists of antagonists of the neurotrophin NGF. NGF binding to its high-affinity receptor tyrosine kinase receptor A (TrkA) in the dorsal root ganglion induces a signal cascade that results in the release of calcitonin gene-related peptide, substance $\mathrm{P}$, and brainderived neurotrophic factor. Beside nerves, cells such as keratinocytes express NGF. During inflammation, mast cells and macrophages serve as additional sources of NGF. Pfizer Inc (New York, NY, USA) has developed a monoclonal antibody (tanezumab) against NGF. Clinical trials for the treatment of knee pain due to OA have been stopped recently. However, clinical trials for other pain conditions are ongoing. Since the treatment of large groups of patients with monoclonal antibodies is costly, a 
vaccination against NGF (NGF-Q $\beta$ ) was developed in Zeilhofer's lab. Vaccinated mice in a model of inflammatory pain recovered faster than control animals. Furthermore, vaccination reduced thermal hyperalgesia in a mouse model of CIA. Side effects on peptidergic sensory nerve fibers were not observed. Furthermore, no undesired effects concerning the integrity of the bloodbrain barrier were found.

\section{Better understanding the hidden face of osteoarthritis Jean-Pierre Pelletier, Montreal, QC, Canada}

In his talk, Jean-Pierre Pelletier pointed out the necessity of the development of disease-modifying drugs for the treatment of OA (DMOADs). So far, no DMOADs have been approved by the US Food and Drug Administration, and current therapies for OA are directed toward controlling symptoms, maintaining function, and reducing further joint damage. A challenging issue in the development of DMOADs is the design of clinical trials. First of all, a careful selection of patients is important since age, gender, body mass index, and other factors have been reported to have an impact on study outcome. So far, no clear guidelines for inclusion criteria for patients exist. Other important aspects are the method of determination of disease progression and the selection of target joints. So far, the evaluation of symptoms in DMOAD trials is the same as in short-term trials. Pelletier suggested focusing on the global evaluation of structural changes in several joints instead of measuring a reduction of pain on the visual analogue scale. Furthermore, he recommended the use of quantitative magnetic resonance imaging (qMRI) instead of $\mathrm{x}$-ray technology for the measurement of structural changes in the joints. In 2009, Raynauld and colleagues published the first multicenter clinical trial study using qMRI (Raynauld JP et al., Ann Rheum Dis, 2009). Pelletier advised the recording of total joint replacements after 6 years of follow-up; these data, which would serve as an adequate readout for DMOAD trials, can be easily collected by means of questionnaires. So far, no international guidelines for DMOAD clinical trials exist.

\section{Do regulatory $T$ cells control the switch from acute to chronic arthritis? \\ Thomas Kamradt, Jena, Germany}

In his presentation, Thomas Kamradt pointed out the role of regulatory $\mathrm{T}$ cells (Tregs) in the switch from acute to chronic inflammatory diseases. Tregs are $\mathrm{CD} 25^{+} \mathrm{CD} 4^{+}$ $\mathrm{T}$ cells that express the transcription factor FoxP3. They are generated in the thymus as well as in the periphery from naive $\mathrm{T}$ cells. The exact mechanism of Treg action is unclear. An increased number of Tregs was described in patients with arthritis. On the other hand, in mouse models of CIA and antigen-induced arthritis, Tregs were shown to delay the onset and downregulate the severity of the disease. Hence, Tregs have no effects in models of prostaglandine-induced arthritis. Kamradt reported that immunization of genetically unaltered mice with the ubiquitously expressed glycolytic enzyme glucose-6phosphate isomerase (G6PI) induced arthritis within 9 days. This was a $\mathrm{T}$ cell-dependent disease since depletion of $\mathrm{CD}^{+}$cells prevented and cured G6PI-induced arthritis. G6PI-induced arthritis was monophasic and self-limiting. Induction of a second round of arthritis by repeated G6PI immunization failed. However, transient depletion of $\mathrm{CD}_{25} 5^{+}$cells increased the number of G6PIactivatd $\mathrm{CD}^{+}{ }^{+} \mathrm{CD} 154^{+}$Th cells and caused chronic arthritis. These $\mathrm{CD} 4^{+} \mathrm{CD} 154^{+} \mathrm{Th}$ cells were polyfunctional since they were capable of producing up to five cytokines in parallel upon $\mathrm{CD}_{25} 5^{+}$cell depletion. The mechanism of how an early and transient depletion of Tregs results in chronic destructive arthritis remains to be determined. Kamradt suggested the possibility that newly evolving Tregs in late arthritis are insufficient. Alternatively, an early instruction of effector cells by dysregulated $\mathrm{T}$ cells is possible. Furthermore, direct interaction of Tregs with effector cells might be possible. Polyfunctional T cells might also be relevant in autoimmunity in patients, and Tregs might play an important role in the preclinical phase of arthritis.

\section{Novel epigenetic strategies for autoimmune disease and cancer \\ Wesley Brooks, Tampa, FL, USA}

Wesley Brooks pointed out the role of the loss of epigenetic control in the development of autoimmune diseases. The female predominance of diseases such as multiple sclerosis, RA, lupus erythematosus, and Sjörgren syndrome suggests the involvement of hormones, $\mathrm{X}$ chromosomes, and the $\mathrm{X}$-chromosomal inactivation process. Early in embryogenesis, the second $\mathrm{X}$ chromosome in female cells undergoes inactivation in order to achieve dosage compensation. Approximately $85 \%$ of the genes on the inactivated $\mathrm{X}$ chromosome $(\mathrm{Xi})$ undergo inactivation. Owing to its perinuclear localization, the late replication, hypermethylation, and the limited access for DNA repair, the $\mathrm{Xi}$ is especially vulnerable to DNA damage. DNA mutations could interfere with the $\mathrm{X}$ inactivation process and then could become reactivated in daughter cells, resulting in a loss of epigenetic control. An interesting region on the short arm of the $\mathrm{X}$ chromosome is Xp22.1, which encodes for enzymes involved in the polyamine synthesis (namely spermine synthase and spermine/spermidine N1-acetyltransferase). Many agents, including estrogens, UV light, and viral infections, can induce polyamine synthesis. Increased polyamine synthesis could impact chromatin methylation and epigenetic control because of the increased needs for 
$\mathrm{S}$-adenosylmethionine, which is also used as a substrate for DNA methylation. Furthermore, polyamines can stabilize Z-DNA and alter gene transcription. Abnormal RNA polymerase III activity and overexpression of pseudogenes, such as Alu and LINE L1 sequences, have been described. Brooks suggested the enzymes ornithine decarboxylase and spermine/spermidine N1-acetyltransferase as suitable new targets for drug discovery.

ASIA: a new syndrome of autoimmune diseases induced by adjuvants

Yehuda Shoenfeld, Tal-Hashomer, Israel

In his presentation, Yehuda Shoenfeld discussed an autoimmune disease called 'autoimmune syndrome induced by adjuvant' (ASIA). Autoimmune diseases have multifactorial causes, such as genetic predisposition, immunodeficiency, hormones, and environmental factors, including adjuvants. Adjuvants, the foremost of which is aluminum, are widely used in vaccines to stimulate the immune system. Although Shoenfeld did not want to doubt the beneficial effects of vaccines, he referred to numerous studies and case reports documenting that vaccination of naive subjects can lead to autoimmunity. Causal relationships between diphtheria-tetanus-pertussis vaccination and measles-mumps-rubella (MMR) vaccination with arthritis have been described. Furthermore, a link between MMR and autoimmune thrombocytopenia has been reported. Whereas early adverse events of vaccinations (for example, fever or local reactions at the site of injection) are easily documented, the major difficulty of diagnosing ASIA is the lack of temporal association between the vaccination and the incidence of symptoms, and this lack is due to long latency periods. Moreover, the criteria for causality are not well defined, and unlike efficacy, safety cannot be measured directly. Animal models can be useful for studying the interrelation between vaccination and autoimmune phenomena.

Some vaccinations use silicone as an adjuvant. The wide use of silicone in breast operations led to many symptomatic cases of autoimmune disease. In some cases, the removal of silicone was sufficient for recovery of the patients. Shoenfeld concluded that the association between vaccination and autoimmunity raises many clinical, methodological, and ethical questions and that there is a need for increasing vaccine safety and identifying possible risk factors for the incidence of ASIA.

\section{Abbreviations}

ANCA, antibodies to citrullinated protein antigen; APC, antigen-presenting cell; ASIA, autoimmune syndrome induced by adjuvant; CARM1, coactivatorassociated arginine methyltransferase; $\mathrm{CDC}$, conventional dendritic cell; $\mathrm{CIA}$, collagen-induced arthritis; $C R$, caloric restriction; DAMP, danger-associated molecular pattern; DARPin, designed ankyrin repeat protein; DC, dendritic cell; DMOAD, disease-modifying osteoarthritis drug; DMT2, type 2 diabetes mellitus; DTH, delayed-type hypersensitivity; EAE, experimental autoimmune encephalomyelitis; GARN, Global Arthritis Research Network; G6PI, glucose-6phosphate isomerase; hMSC, human mesenchymal stem cell; hTERT, human telomerase reverse transcriptase; hTNFtg, human tumor necrosis factor transgenic; IFN, interferon; IL, interleukin; LTi, lymphoid tissue-inducer; MHC, major histocompatibility complex; miRNA, microRNA; MMR, measles-mumpsrubella; MSU, monosodium urate; MZ, monozygotic; Nav, Voltage-gated sodium channel; NF-kB, nuclear factor-kappa-B; NGF, nerve growth factor; NLR, NOD-like receptor; OA, osteoarthritis; PAMP, pathogen-associated molecular pattern; PARP-1, poly (ADP-ribose) polymerase-1; PRMT1, protein arginine methyltransferase 1; PrPsc, scrapie prion protein isoform; qMRI, quantitative magnetic resonance imaging; RA, rheumatoid arthritis; ROS, reactive oxygen species; SCID, severe combined immunodeficient; SE, shared epitope; SF, synovial fibroblast; SGC, Structural Genomics Consortium; SLE, systemic lupus erythematosus; TGF- $\beta$, transforming growth factor-beta; $T N F$, tumor necrosis factor; Treg, regulatory T cell; TRPV1, transient receptor potential cation channel subfamily $\vee$ member $1 ; T X N I P$, thioredoxin-interacting protein; Xi, inactivated $X$ chromosome.

Competing interests

The authors declare that they have no competing interests.

Note

The 7th meeting of GARN was held from 7 to 10 October 2010 at the Hotel Renaissance in Zurich, Switzerland. Sessions 1 to 3 were held on 8 October, and Sessions 4 to 6 on the following day.

\section{Acknowledgments}

The 7th meeting of GARN was supported by exclusive unrestricted educational grants from Pfizer Inc (New York, NY, USA), Roche (Basel, Switzerland), and Wyeth Pharmaceuticals (Madison, NJ, USA).

Published: 23 August 2011

doi:10.1186/ar3340

Cite this article as: Brock M, et al.: 7th meeting of the Global Arthritis Research Network. Arthritis Research \& Therapy 2011, 13:303. 